ISSN: $2219-8229$

E-ISSN: 2224-0136

Founder: Academic Publishing House Researcher

DOI: $10.13187 /$ issn. $2219-8229$

Has been issued since 2010 .

European Researcher. International Multidisciplinary Journal

UDC 620.92, 537, 534.121.1

\title{
«Green» Energy Harvesting by Means of Piezoflexogeneration from Vibration or Similar Processes
}

\author{
1Timofey G. Lupeiko \\ ${ }^{2}$ Boris S. Medvedev \\ ${ }^{3}$ Mikhail I. Evdokimov
}

1. Southern federal university, chemistry department, Russian Federation

344090, Rostov-on-Don, 7, R. Zorge street

Dr. (chemistry), Professor

E-mail: lupeiko@sfedu.ru

2. Southern federal university, Russian Federation

344090, Rostov-on-Don, 7, R. Zorge street

$\mathrm{PhD}$ (chemistry), associate professor

E-mail: oursov37@yandex.ru

3. South federal university, Russian Federation

344090, Rostov-on-Don 7, R. Zorge str.

senior laboratory assistant, bachelor of material science

E-mail: michalych91@mail.ru

Abstract. The piezoelectric systems of electric energy harvesting with an adaptive lowfrequency resonance are developed. These systems allowed to obtain electricity from low-frequency vibration. The availability of their application for adaptation to other periodic processes including pedestrians and vehicles movement is shown.

Keywords: Energy harvesting systems; piezogenerator; alternative energy; "green" electric energy from vibration.

Introduction. The modern advance in the sphere of energetics is taking place under conditions when conventional basic energy sources have been developed and the most large-scale resource associated with hydrocarbon raw materials is objectively restricted. As a result the search for new alternative energy sources and proper ways of their adaptation is of great interest. Therefore, the solutions oriented on new energy source adaptation that will allow obtaining practically "green" electric energy are especially important. Vibration is one of such sources. It follows various anthropogenic and natural processes and is lost being only an additional ecological load for the environment. However the question of considerable energy obtaining and harvesting from the most common low-force vibration all recent achievements in this sphere [1-5] taken into account is still under discussion.

Materials and Methods. The new energy harvesting system development and usage results are given in the article, piezoelectric transducers which generate electric charge under mechanical effect being applied. At present this is the way of obtaining electric energy in the number of mainstream devices (piezoelectric lighters, electroshocks, etc.) with ceramic piezoelements functioning in the direct "compression-stretching" mode. These piezoelements are also suggested for systems harvesting energy from moving vehicles [6]. However, while these 
devices are operating, piezoelements undergo high mechanical and electrical loads. This lowers their functionality and hampers their applying in energy harvesting systems where they are supposed to function for long periods under such strict conditions. In addition, when using these element containing batteries a serious challenge is to synchronize separate elements' work by time and phase and to correlate the obtained voltages $(\mathrm{kV})$ with outer electric circuits. The decrease of force impact on these ceramic piezoelectric elements up to order of units $(\mathrm{N})$ force level leads to their piezogeneration effectiveness decrease so that it becomes impossible to gain electric energy from low-force vibration and similar processes.

We have developed new type active piezomaterials and highly effective transducers made on their base as well as created new energy harvesting systems where these transducers are applied [79].

We managed to increase piezogeneration effectiveness from low-force impacts, which allowed us to develop the functioning model producing electricity from vibration [10]. While solving this problem we proceeded from the fact that piezogeneration effectiveness, when a piezoelectric element was affected mechanically, is mainly determined by its piezoelectric constants, dielectric permittivity and elastic compliance. The function of two first properties is evident, because electric energy (W) generated by the piezoelectric element under the force $(F)$ impact, for example, in its polarization coinciding direction, is determined by relation:

$$
\mathrm{W}=\left(\mathrm{d}_{33} \cdot \mathrm{F}\right)^{2} / 2 \cdot \mathrm{C} \text {, }
$$

where $d_{33}$ - longitudinal vibration piezoelectric constant and $\mathrm{C}$ - capacity linear to its dielectric permittivity.

The elastic compliance function is essentially different. It determines and limits the mechanical energy which piezoelectric element, when affected by force, accepts for transformation. This energy equals the product of the force affecting the piezoelement by its linear size change in the direction of this force impact. It is this size change that is determined by its elastic compliance.

Our solution is based on applying flexible piezoelements with high elastic compliance. Their linear size change as compared to that of ceramic elements which work in direct "compressionstretching" mode when affected by one and the same force is ten thousands of times more. It leads to proportional flexible element-generated electric energy increase (table 1) and allows obtaining ten thousands of times higher energy from comparatively weak mechanical effects. The open circuit voltage generated by these elements can be up to $30 \mathrm{~V}$.

Table 1. The values of electrical energy generated by flexible piezoelectric element (new solution) and typical ceramic piezoelectric element operating in the direct "compression-stretching" mode (analogue)

\begin{tabular}{|l|l|l|}
\hline \multirow{2}{*}{ Force, $\mathbf{N}$} & \multicolumn{2}{|c|}{ One element energy, $\mathbf{\mu J}$} \\
\cline { 2 - 3 } & New solution & Analog \\
\hline 1 & 60 & $\mathbf{0 . 0 0 1 5}$ \\
\hline 2 & 120 & $\mathbf{0 . 0 0 6 0}$ \\
\hline 3 & 200 & $\mathbf{0 . 0 1 3 5}$ \\
\hline 5 & 205 & $\mathbf{0 . 0 3 7 5}$ \\
\hline
\end{tabular}

According to Table 1, the energy generated by flexible piezoelectric elements (when force load grows) firstly increases in direct ratio and then practically remains invariable. This fact makes it reasonable to limit force impact level when the elements are used as energy generators. It is also important that this limitation level is significantly lower than force load values, maximum permissible for these piezoelectric elements. As a result, the used flexible element effective work conditions appear to be rather comfortable. The testing showed that if optimal work conditions are kept the elements do not lose their performances after $0,5 \times 10^{9}$ operations (the persistent running mode under conditions of energy generation from $50 \mathrm{~Hz}$ frequency vibration with $2.5 \mathrm{~N}$ force load for 120 days). 
When flexible piezoelectric elements are used as energy generators there is one more important opportunity for their work optimization. It is connected with the fact that on the base of flexible piezoelectric elements it is possible to construct the mechanical schemes with the given low-frequency resonance. As it can be seen from fig. 1 the vibration frequency adapted resonance systems allow significant energy generation effectiveness increasing.

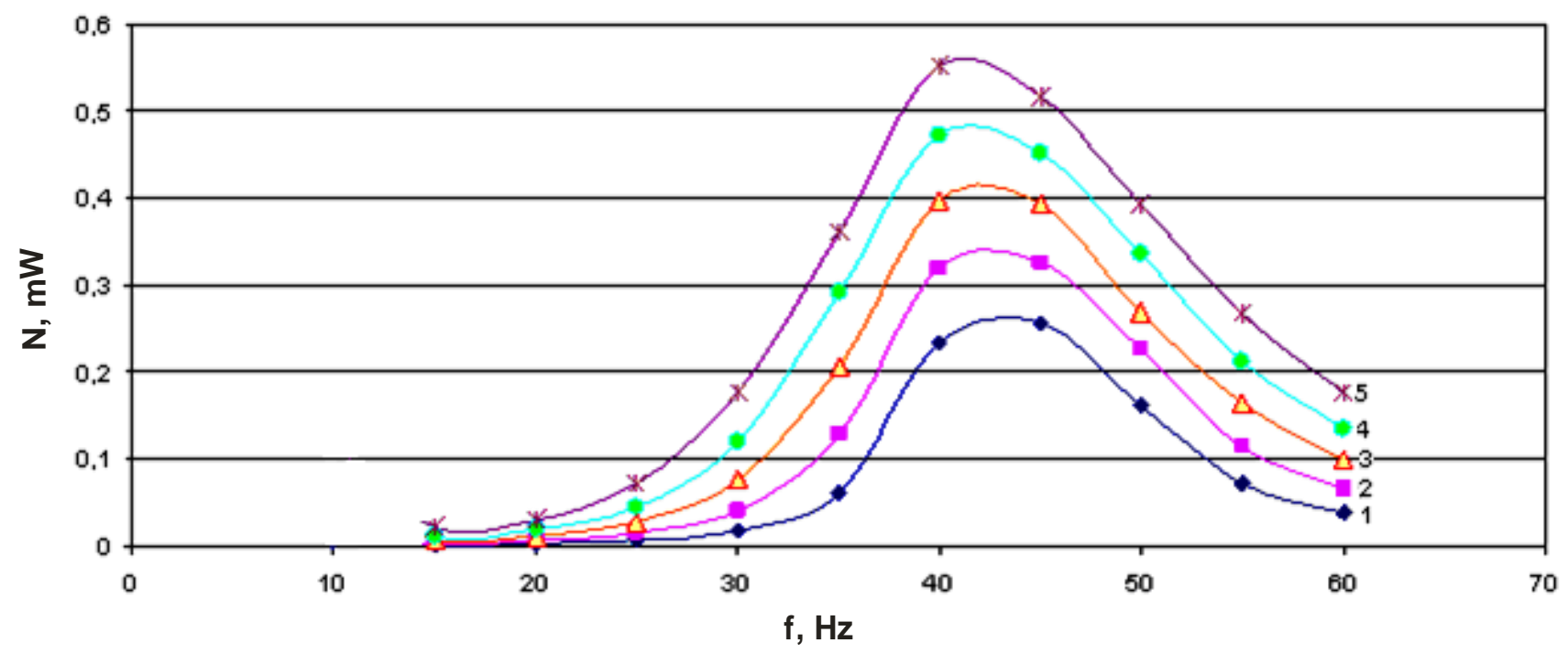

Fig. 1. The dependence of energy piezogeneration power obtained from flexible piezoelectric elements on vibration frequency and amplitude (curves $1 ; 2 ; 3 ; 4$ and 5 correspond to vibration amplitude $0.3 ; 0.4 ; 0.5 ; 0.6$ и 0.7 g respectively).

With regard to permissible force loads on flexible piezoelectric elements and their elastic properties the possible resonance system frequency is limited up to $100 \mathrm{~Hz}$. This leads to new opportunities to obtain energy from new sources including various types of low-frequency natural and anthropogenic vibrations as well as similar processes.

Discussion. It is also important that the number of potentially prospective processes which can become the energy obtaining source with flexible piezoelectric element use include wave processes as well as periodic effects from pedestrian and vehicle movement. They can be also regarded as low-frequency vibration sources. Taking into account the limit for flexible piezoelectric element force impact permitted level to adapt these high-power sources we should develop multielement energy harvesting systems where outer force impact is distributed among separate flexible piezoelectric elements and does not exceed their permitted values.

The example of such solution is a functioning five-element generator that we prepared [10] which develops power up to $3 \mathrm{~mW}$ when vibration is transformed with the frequency of $30 \mathrm{~Hz}$, acceleration of $1 \mathrm{~g}$ and general force load of $10 \mathrm{~N}$.

Using the opportunity to create multiunit flexible element piezoelectric generators it is possible to drastically increase energy harvesting system power and provide adaptation of practically any mechanical loads.

It is noteworthy that the voltages generated by new energy harvesting systems are essentially lower than kilovolt voltages that are obtained in ceramic element-based piezoelectric generators that work in direct "compression-stretching" mode. This allows usage of ordinary batteries and condensers to accumulate the harvested energy. Fig. 2 shows a typical curve for $10 \mathrm{mF}$ capacity condenser charging with one flexible piezoelectric element generating energy from vibration with $30 \mathrm{~Hz}$ frequency and $0.7 \mathrm{~g}$ amplitude at $0.25 \mathrm{~N}$ load (for the obtained alternating voltage rectification a low-volt diode bridge was used). The same figure shows the dynamics of this process optimization in recent years. 


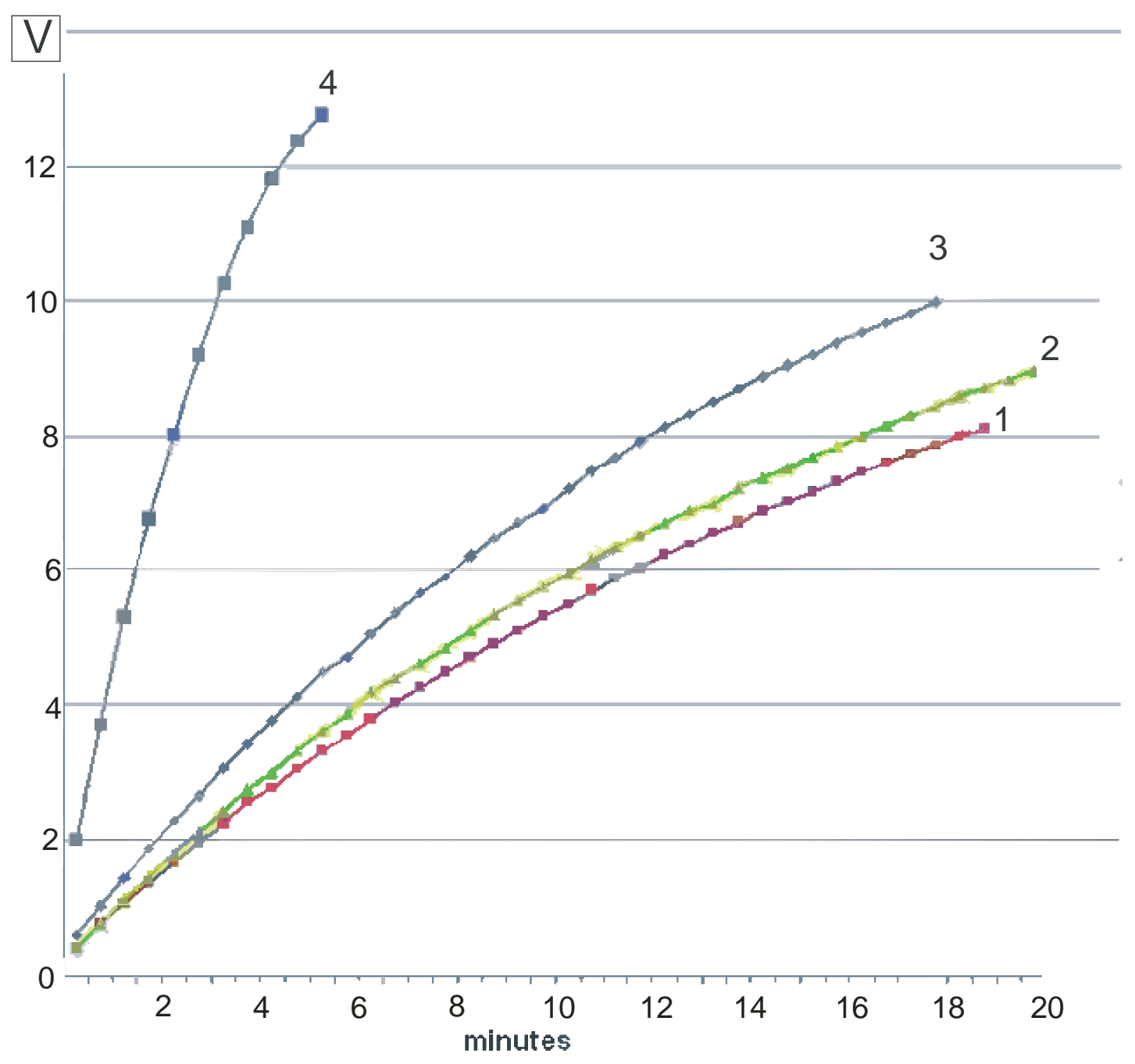

Fig.2. The rate of $10 \mathrm{mF}$ capacity condenser charging with one flexible piezoelectric element generating energy from vibration (curves 1, 2, 3 and 4 correspond to the results obtained in 2010, 2011, 2012 and 2013 respectively)

It is clear from the figure that during the last three years it became possible to get a 6-time increase in the effectiveness of electric energy generation from one and the same mechanical source only for account of active element optimization.

Based on appropriate structural solutions in prospective it is possible to obtain:

- milliwatts from low-frequency vibration and similar processes;

- watts from pedestrian moving along sidewalks, underground stations, etc.;

- kilowatts from vehicle moving.

In general, it seems interesting to create Energy Harvesting Cluster which will allow working in quite a number of prospective directions (fig. 3). 


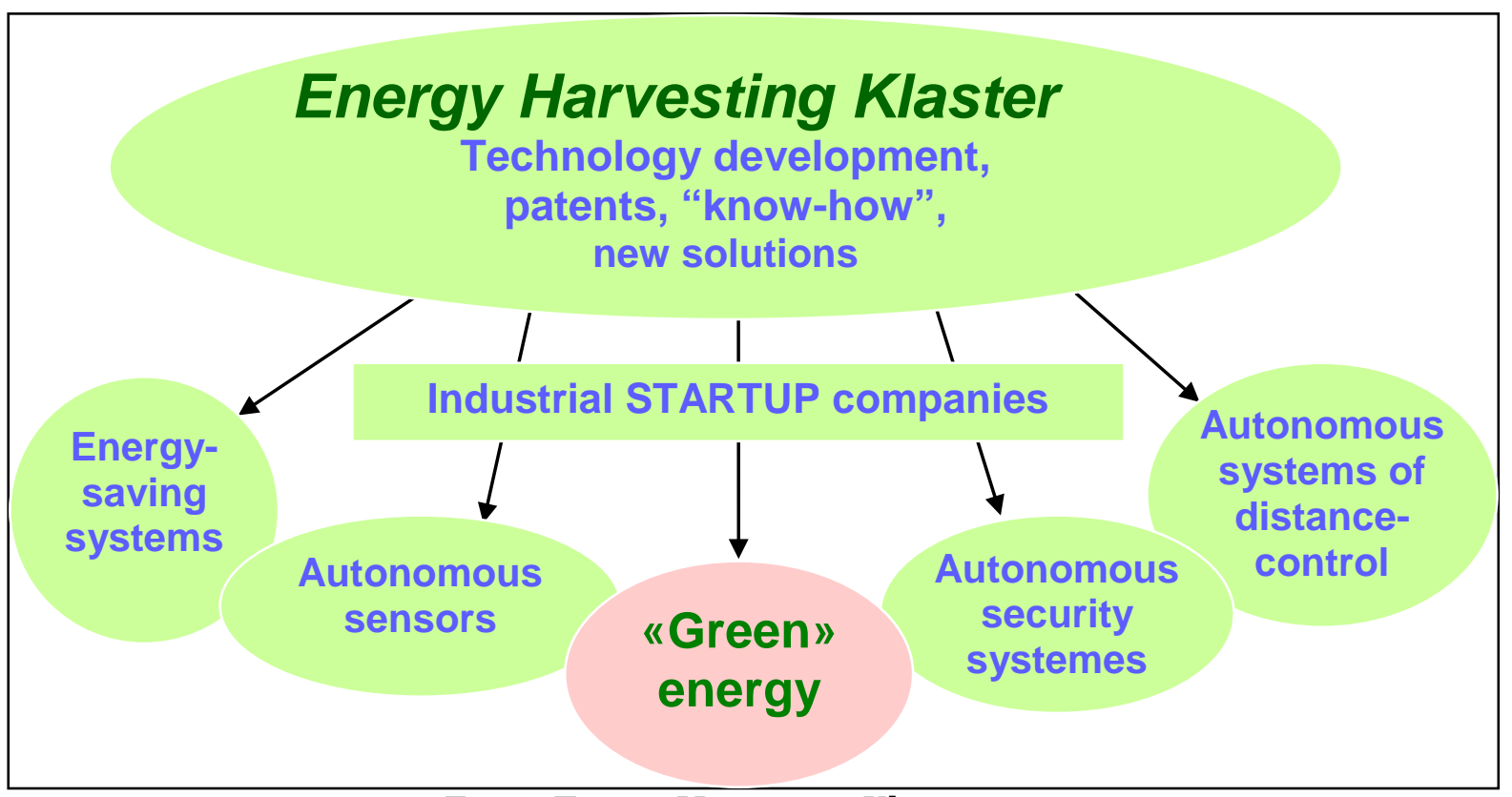

Fig. 3. Energy Harvesting Klaster

\section{Conclusion}

A new solution for systems harvesting energy from low-frequency vibration and similar processes was obtained. It is based on:

- the usage of high elastic compliance flexible piezoelectric elements;

- the application of energy harvesting mechanical systems with adaptive low-frequency resonance;

- the opportunity to create multiunit piezoelectric generators;

- the combination of electric energy generation with the favorable conditions of its accumulation.

\section{Referencies:}

1. Guyomar, D. Towards Energy harvesting using active materials and conversion improvement by nonlinear processing. / D. Guyomar, A. Badel, E. Lefeuvre, and C. Richard // IEEE Transactions on Ultrasonics Ferroelectrics and Frequency Control. 2005. Vol. 52. I. 4. P. 584-595.

2. Lefeuvre, E. A comparison between several vibration-powered piezoelectric generators for standalone systems. / E. Lefeuvre, A. Badel, C. Richard, L. Petit and D. Guyomar // Sensors and Actuators A. 2006. V. 126, № 2, P. 405-416.

3. Hongduo, Z. A comparative analysis of piezoelectric transducers for harvesting energy from asphalt pavement. / Z. Hongduo, Jianming L. and J ian Y. // J ournal of the Ceramic Sosiety of Japan. 2012. V. 120. № 8. P. 317-323.

4. Lefeuvre, E. Optimization of piezoelectric electrical generators powered by random vibrations. / E. Lefeuvre, A. Badel, C. Richard, L. Petit, D. Guyomar // Symposium on Design, Test, Integration and Packaging of MEMS/ MOEMS. Stresa, Italy, 26-28 April 2006.

5. Wang, Z. L. Progress in nanogenerators for portable electronics / Z. L. Wang, G. Zhu, Y. Yang, S. Wang, C. Pan // Materials Today. 2012. V. 15. № 12. P. 532-544.

6. Patent US 2005/0127677 US 20050127677 A1, 25.01.2004. Luttrull J. K. Roadway generating electrical power by incorporating piezoelectric materials / Patent US 10/ 995,991.

7. Lupeiko, T. G. Old and New Problems in Piezoelectric Materials Research: Materials with high Hydrostatic Sensitivity / T. G. Lupeiko, S. S. Lopatin // Inorganic Materials. 2004. V. 40. № 1. P. 19-32.

8. Parinov, I. A. Ferroelectrics and Superconductors: Properties and Applications. Chapter 8. Computer modeling of resonance characteristics of the piezoelectric cylindrical and bimorph transducers In Ferroelectrics and Superconductors: Properties and Applications / T.G. Lupeiko, 
A.V. Soloviev, B.S. Medvedev, A.S. Pahomov, M.P. Petrov, V.S. Chernov and D.S. Nasonova. N-Y.: Nova Science Publishers, 2011. P. 255-272.

9. Lisnevskaya, I. V. PZT-36/ $\mathrm{NiCO}_{0.02} \mathrm{Cu}_{0.02} \mathrm{Mn}_{0.1} \mathrm{Fe}_{1.8} \mathrm{O}_{4}$ Magnetoelectric Rod Composites with 1-3, 3-1, and 1-1 connectivity / I.V. Lisnevskaya, T. G. Lupeiko, I. A. Bobrova, A. V. Petrova // Inorganic materials. 2012. V. 48. № 4. P. 410-415.

10. Lupeyko T.G. Showpiece: "Green energy is a high fleksopiezogeneration", presented at the $\mathrm{X}$ Moscow International Salon of Innovations and Investments. 2010 (awarded a gold medal). 